\title{
UNIVERSIDADES PRIVADAS, ¿UN SUBSISTEMA \\ DIFERENTE?: CÓMO DESARROLLAN LOS ESTUDIANTES \\ SUS PREFERENCIAS HACIA LA UNIVERSIDAD
}

\section{PRIVATE UNIVERSITIES, A DIFFERENT SUBSYSTEM?: HOW THE STUDENTS DEVELOP THEIR PREFERENCES TOWARDS THE UNIVERSITY}

\author{
AUTORES \\ Joan Francesc Fondevila Gascón: Universitat Abat Oliba CEU (UAO). Barcelona (España). \\ jfondevilag@uao.es \\ Josep Lluís del Olmo Arriaga: Universitat Abat Oliba CEU (UAO). Barcelona (España). \\ jlolmo@uao.es \\ Javier Sierra Sánchez : Universitat Abat Oliba CEU (UAO). Barcelona (España). \\ jsierras@uao.es
}

\section{RESUMEN}

Existe un creciente interés en saber cómo los estudiantes desarrollan sus preferencias hacia las universidades y qué les influye en su percepción de tales instituciones. El foco de esta investigación recae en conocer qué factores tienen en cuenta los aspirantes para seleccionar un grado. Este trabajo también tendrá en consideración en qué forma los factores de elección de universidad figuran en la mente de los estudiantes. En este contexto, una encuesta sobre un conjunto representativo de estudiantes del primer curso (primer semestre) ayuda a dar luz a tal estudio. La muestra es de la Universitat Abat Oliba CEU y está dirigida a revelar qué visión tienen los estudiantes con relación a las universidades privadas. Dadas las particularidades de esta universidad, el estudio 
provee datos útiles sobre la formación de preferencias de los alumnos hacia las universidades. La información obtenida en este estudio se puede utilizar como herramienta a la hora de la toma de decisión por parte de la institución para conocer a los estudiantes interesados, y también para poder proporcionar a los interesados más información y más adecuada para que éstos puedan realizar su elección.

\section{PALABRAS CLAVE}

Factores de Elección - Estudiantes - Universidad Privada - Marketing Estratégico Universitario - Instituciones

\section{ABSTRACT}

There exists an increasing interest to know how the students develop their preferences towards the universities and what influences in their perception of such institutions. The area of this investigation relapses in knowing what factors the aspirants bear in mind to select a degree. This work will have in consideration in what it forms the factors of election of university appear in the mind of the students. In this context, a survey on a representative set of students of the first course (the first semester) helps to give light to such a study. The sample is of the Universitat Abat Oliba CEU and there is directed to reveal what vision the students have with relation to the private universities. Given the particularities of this university, the study provides useful data about the formation of preferences of the pupils towards the universities. The information obtained in this study can be in use as tool at the moment of the capture of decision on the part of the institution for knowing the interested students, and also to be able to provide more information and more suitable to the interested parties in order that these could realize his choice. 


\section{KEY WORDS}

Factors of Choice - Students - Private University - Strategic University Marketing Institutions

\section{ÍNDICE}

\section{^ 1. PRESENTACIÓN \\ 2. MARCO TEÓRICO}

2.1 Revisión bibliográfica sobre los factores de elección de Universidad 3. ANÁLISIS EMPÍRICO

3.1 Objetivos y metodología de la investigación

3.2 Resultados de la investigación

\section{CONCLUSIONES Y FUTURAS LÍNEAS DE INVESTIGACIÓN}

\section{PRESENTACIÓN}

Las investigaciones relativas al acceso a los estudios universitarios son numerosas y enfocadas sobre todo a conocer las demandas de estudios y/o carreras que efectúa el alumnado preuniversitario. Todos parecen coincidir en que la elección de estudios universitarios, uno de los hechos más significativos en la vida de una persona, se realiza en condiciones no idóneas, como, por ejemplo, falta de información, ausencia de servicios de orientación educativa, condicionantes por ofertas de estudios en el lugar de residencia o limitaciones de los centros o 'númerus clausus' (De Miguel et alii., 1994: 16) .

En cambio, la investigación sobre las opciones institucionales, ampliamente desarrollada en otros contextos nacionales, ha sido escasa en Cataluña y, en general, en 
el resto de España. En otros países, especialmente en los anglosajones, los estudios sobre la elección de universidad se han multiplicado a partir de la implementación, entre otras, de políticas que pretenden estimular la competencia entre las instituciones a través de medidas como la financiación de la demanda, la evaluación de los resultados de las universidades y el suministro de información a las familias (Del Olmo, 2009).

Para examinar cómo los estudiantes escogen una institución de educación superior, es necesario comprender qué eligen y por qué lo hacen. Además, también puede afectar su decisión final la capacidad que tienen de acceder a la búsqueda de información, asimilar el material y escoger entre alternativas similares. Por ello, se pueden formular las siguientes preguntas:

-¿Cómo escogen los futuros estudiantes universitarios entre diferentes alternativas?

¿¿Qué valoración hacen de las alternativas que tienen a su alcance?

-¿Cómo buscan la información sobre las diferentes opciones?

-¿Cuáles son las influencias en su comportamiento de búsqueda de información?

-¿Cómo valoran la información obtenida?

-¿Qué capacidad tienen de tomar una decisión adecuada sobre un producto tan complejo como una institución de educación superior?

Comprender correctamente el fenómeno de la elección de una institución de educación superior supone determinar no tan solo el porqué sino también el cómo de la decisión (Bowe, Gewirtz y Ball, 1994). El análisis de los factores que influyen en la demanda se hace indispensable, además, si se tienen en cuenta cuestiones como la 
desarticulación existente entre la educación secundaria y superior que impide el acompañamiento de los jóvenes en el tránsito de una a otra etapa de formación y a una equitativa distribución de la información, así como a la progresiva proliferación de instituciones de educación superior, con la consiguiente diversificación y complejidad de la oferta, tanto pública como privada. Ello dificulta la identificación de las posibilidades que ofrece el sistema a los estudiantes.

La investigación en la materia pretende detectar los factores de elección de universidad desde la perspectiva de una institución universitaria de carácter privado como es la Universitat Abat Oliba CEU (Tabla 1), excluyendo el aspecto vocacional (decisión de la carrera elegida). Ello también permite contar con el perfil sociodemográfico del alumnado matriculado en primer curso de Grado de las diferentes titulaciones que componen la oferta formativa de la citada universidad y disponer de elementos relevantes para la planificación estratégica y la toma de decisiones de la institución. El conocimiento de los factores de elección permite sugerir recomendaciones que favorezcan el acceso de los individuos a una institución privada $\mathrm{y}$, por tanto, constituye la base para elaborar de forma más eficaz estrategias comerciales que incluyan una oferta atractiva para estimular la demanda de productos educativos ofrecidos por este perfil de universidad.

Tabla 1: Características de la Universitat Abat Oliba CEU

\begin{tabular}{|l|l|}
\hline Año de creación & 2003 \\
\hline Estudios de Grado & $\begin{array}{l}\text { Derecho, Dirección de Empresas, Marketing y } \\
\text { Dirección Comercial, Magisterio de Educación } \\
\text { Infantil, Magisterio de Educación Primaria, } \\
\text { Publicidad y RRPP, Periodismo y Psicología }\end{array}$ \\
\hline Titularidad/carácter & Privada, sin ánimo de lucro \\
\hline Criterios de selección & $\begin{array}{l}\text { Superar los niveles previos del sistema educativo } \\
\text { y pruebas de admisión propias de la universidad }\end{array}$ \\
\hline Coste de los estudios (Matrícula anual) & Entre 5.880 y 8.220 euros \\
\hline
\end{tabular}

Fuente: Elaboración propia. 
En cualquier caso, la decisión que toma el alumnado preuniversitario no es totalmente libre, dado que está condicionada por diversas circunstancias, como los estudios previos cursados, la modalidad de bachillerato escogida, el expediente académico de los estudios previos, la limitación de plazas públicas y privadas ofertadas, el nivel educativo de los padres, los costes directos y de oportunidad de la universidad, el sistema de becas o proximidad del centro, entre otras (Del Olmo, 2009).

El conocimiento que tienen los estudiantes sobre sus posibilidades de acceder a unos estudios concretos y a una determinada institución de educación superior puede permitir intuir el efecto de autocensura (Cañabate, 1998: 240), a través del cual se puede establecer una diferenciación entre patrones de elección de universidad manifiestos e ideales. Los primeros son el resultado de las elecciones que los solicitantes emiten considerando, por una parte, sus preferencias ideales (si no existiesen dificultades para acceder tanto a los estudios que se desean como a la universidad) y, por otra, sus posibilidades reales de acceso.

\section{MARCO TEÓRICO}

\subsection{Revisión bibliográfica sobre los factores de elección de Universidad}

Los criterios de elección se pueden clasificar de diferente manera según el nivel de motivación para buscar la información, la capacidad para la toma de decisiones del estudiante, así como el nivel y el tipo de búsqueda de información llevada a cabo por el futuro alumno universitario.

Los estudios sobre la elección de universidad se han orientado sobre todo hacia las expectativas de los estudiantes, medidas generalmente de manera ex-post, y 
relacionadas, por ejemplo, con la imagen institucional (Belanger, Mount y Wilson, 2002), las preferencias (Soutar y Turner, 2002; Holdsworth y Nind, 2005) o las motivaciones (Cuestas, Fenollar y Román, 2006). Por ello, los procesos de elección como etapa de compra son relevantes aunque difícilmente generalizables (Shanka, Quintal y Taylor, 2005), lo que acredita la alta influencia del entorno socioeconómico de la demanda universitaria (Rahona, 2006).

Por ello, conocer los criterios por los que los estudiantes seleccionan una universidad es básico para posicionar a dicha institución en el nuevo mercado competitivo (Fielder, Milton y Motes, 1993; Maaringe, 2006; Luque y Del Barrio, 2007; Gallarza, Fayos, Servera y Arteaga, 2008). La importancia de estos criterios puede variar en función del tipo de estudiante. Los más relevantes para la opción educativa son los siguientes (Jackson, 1986; Cabrera y La Nasa, 2000; Looker y Lowe, 2001):

-La capacidad y preparación académica.

-El interés en el área de estudio.

-La experiencia familiar en la formación universitaria ${ }^{1}$.

-El producto institucional: los programas académicos y no académicos, la reputación de la universidad, la normativa académica, los criterios de admisión, la tipología de alumnado de la universidad y el profesorado.

-Los servicios y los procesos: la disponibilidad de plazas ofertadas, la flexibilidad en la oferta de programas académicos, la variedad en la oferta de programas académicos,

\footnotetext{
1 Algunos trabajos (Fernández López et al. 2001; Jiménez y Salas, 2000; Obis y Rialp, 1996) argumentan que una variable importante que influye en la decisión de continuar los estudios universitarios es el nivel educativo de los padres y sus niveles de ingreso.
} 
la accesibilidad a los programas académicos, las instalaciones, las actividades extraacadémicas, las actividades sociales, la disponibilidad de alojamiento, los programas practicum y bolsa de trabajo, el seguimiento autorizado de los programas de estudio y la seguridad personal de los estudiantes.

-Los costes y la ayuda financiera asociados a la asistencia a la universidad.

-El estatus socio-económico.

-La ubicación de la universidad.

El servicio de formación universitario es multidimensional en esencia (Gallarza et al., 2008), agrupando elementos cognitivos (por ejemplo, oferta amplia o estrecha de titulaciones) y afectivos (ambiente en el campus), individuales (uso de plataformas de formación) y sociales (compañerismo), o también endógenos (reputación) y exógenos (localización). Esta componente multidimensional constituye una dificultad añadida a la gestión de la universidad, pero también la fuente de obtención de ventajas competitivas en la combinación sinérgica de estos elementos, por lo que resulta necesario conocer de forma periódica su valoración por parte del alumnado universitario. Por ello, el estudio empírico que se presenta a continuación constituye una contribución en este sentido.

\section{ANÁLISIS EMPÍRICO}

\subsection{Objetivos y metodología de la investigación}

Tal como se ha indicado al inicio de este artículo, la presente investigación tiene como principal objetivo mejorar el conocimiento sobre las opiniones y preferencias del alumnado de la Universitat Abat Oliba CEU, así como las motivaciones y actitudes que manifiesta en relación a estas preferencias. Para el estudio empírico, este objetivo global 
se concreta en los siguientes objetivos específicos, entendidos como cuestiones a investigar (al tratarse de un estudio exploratorio, no se plantean hipótesis formales a contrastar):

-Determinar el origen académico de los alumnos de la Universitat Abat Oliba CEU y valorar la correspondencia de este análisis con los factores de elección de universidad, según las variables incluidas en el estudio.

-Medir la importancia y valoración de los principales factores vinculados a la elección de universidad, y vincular esta valoración con el análisis teórico efectuado.

En la investigación se ha analizado una muestra representativa del total de alumnos inscritos en primer curso de Grado de la Universitat Abat Oliba CEU. Dado el carácter exploratorio del estudio, el muestreo ha sido no probabilístico, utilizando el muestreo por cuotas, y seleccionando una muestra de conveniencia, aunque respetando la estructura del perfil de estudiantes objeto de estudio, para que no existiesen diferencias por especialidades, ni tampoco desviaciones comparativas (Tabla 2).

Tabla 2: Parámetros básicos de la investigación empírica

\begin{tabular}{|l|l|}
\hline Enfoque de la investigación & Exploratorio y descriptivo \\
\hline Método & Cuantitativo (cuestionario estructurado) \\
\hline Sistema de administración & Entrevista personal \\
\hline Población & $\begin{array}{l}\text { Alumnado de primer curso de Grado de } \\
\text { la Universitat Abat Oliba CEU }\end{array}$ \\
\hline Método de muestreo & Muestreo por cuotas y de conveniencia \\
\hline Fecha del trabajo de campo & Noviembre de 2010 \\
\hline
\end{tabular}

Fuente: Elaboración propia.

2 21,25\% del Grado de Derecho, 12,08\%, del Grado de Dirección de Empresas, 5,48\% del Grado de Marketing y Dirección Comercial, 9,58\% del Grado de Magisterio de Educación Infantil, 12,92 \% del Grado de Magisterio de Educación Primaria, 14,26\% del Grado de Publicidad y RRPP, 7,92\% del Grado de Periodismo y 15,42\% del Grado de Psicología. 
Con la información extraída de la revisión bibliográfica se redactó un cuestionario estructurado que, antes de su lanzamiento, fue sometido a un pre-test con 26 alumnos, en el que subsanó algún problema de interpretación de las preguntas.

El cuestionario definitivo, además de los datos personales, incluía variables sobre motivaciones que medían la influencia que tienen los diferentes atributos de la universidad en el proceso de elección. Para ello, se utilizaron factores relativos a los cuatro instrumentos de marketing de la oferta (servicio ofrecido, precio, promoción y distribución), así como los que hacían referencia a las influencias personales de la familia, escuela o de otras personas que estudiaban o habían estudiado en la universidad, en función del valor que les otorgaba el alumnado, en una escala de Likert de cinco puntos.

Ante los factores de decisión planteados, los estudiantes debían manifestar el nivel de influencia que ejercía cada uno de ellos, oscilando las respuestas desde Ninguna influencia, valor 1, a Mucha influencia, valor 5, con tres niveles intermedios (Poca influencia, valor 2, Alguna influencia, valor 3, y Mucha influencia, valor 4). La escala se aplicó sobre 47 factores, agrupados según los criterios siguientes: conocimiento de la universidad, profesorado, estudiantes, metodología, bolsa de trabajo, servicios, coste y financiación, promoción e información, campus, referencias y otros (Del Olmo, 2009).

El cuestionario fue pasado a los alumnos en el mes de noviembre de 2010 mediante entrevista personal con individuos previamente formados, obteniéndose 239 cuestionarios válidos.

\subsection{Resultados de la investigación}

Al analizar el género y tipo de centro de procedencia del alumnado, se comprueba que éste proviene mayoritariamente de centros de carácter privado/concertado 
$(64,1 \%)$, siendo el porcentaje de mujeres $(79,06 \%)$ sensiblemente superior al de hombres $(20,94 \%)$. Este hecho se explica en parte por la tipología de la oferta formativa de la Universitat Abat Oliba CEU, centrada fundamentalmente en el ámbito de las Ciencias Sociales.

El 51,72\% del total de encuestados son mujeres que provienen de centros de titularidad privada/concertada, mientras que el porcentaje de hombres con este origen es tan sólo del 12,39\%. La procedencia de mujeres de centros públicos es del 27,35\% y la de hombres, del 8,55\%, respecto al total de individuos participantes en la encuesta.

Respecto a los factores de elección, y una vez jerarquizados éstos en el estudio empírico, la Tabla 3 muestra los valores totales obtenidos para cada una de las proposiciones formuladas en la encuesta, ordenadas de mayor a menor influencia. Estos valores, según la escala de Likert, oscilaban entre el Ambiente de estudio $(4,28)$, como valor más representativo y la Dificultad para acceder a una universidad privada $(2,10)$, que fue el atributo menos valorado por la muestra. Los únicos factores que superaron el nivel de Bastante influencia (4) fueron el Ambiente de estudio $(4,28)$, la Accesibilidad del profesorado a los estudiantes $(4,08)$ y la Amabilidad en el trato del personal de la universidad $(4,07)$.

Gráfico 1. Porcentaje de estudiantes matriculados en primer curso de Grado según género y centro de procedencia

Estudiantes vs. Procedencia Académica 


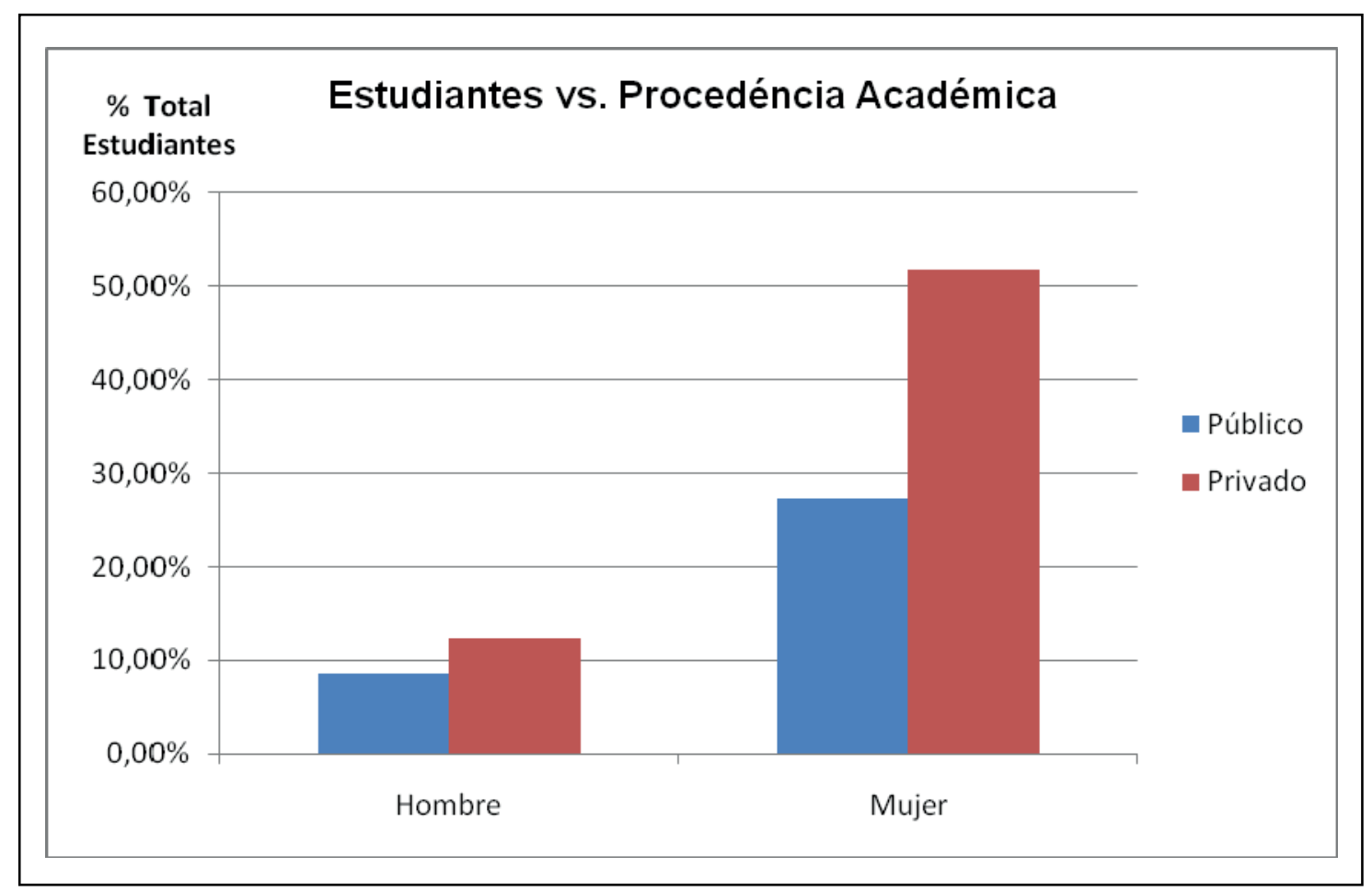

Fuente: Elaboración propia a partir de datos de la encuesta

Tabla 3: Razones para elegir universidad

\begin{tabular}{|c|l|c|c|}
\hline $\begin{array}{c}\text { Orden } \\
\text { secuencial }\end{array}$ & \multicolumn{1}{|c|}{ Factores de Elección } & $\begin{array}{c}\text { Puntuación } \\
\text { hasta 5 }\end{array}$ & $\begin{array}{c}\text { Desviación } \\
\text { Típica }\end{array}$ \\
\hline 1 & Ambiente de estudio & 4,28 & 1,06 \\
\hline 2 & Accesibilidad del profesorado a los estudiantes & 4,08 & 0,86 \\
\hline 3 & Amabilidad en el trato del personal de la universidad & 4,07 & 0,84 \\
\hline 4 & Experiencia profesional de los profesores & 3,96 & 0,73 \\
\hline 5 & Variedad de los grados ofertados & 3,92 & 0,69 \\
\hline 6 & Calidad de la educación & 3,87 & 0,64 \\
\hline 7 & Capacidad pedagógica del profesorado & 3,83 & 0,60 \\
\hline 8 & Calidad del servicio de asesoramiento y atención al & 3,76 & 0,53 \\
\hline 9 & alumnado & 3,75 & 0,52 \\
\hline 10 & Orientación práctica del estudio & 3,69 & 0,47 \\
\hline 11 & Reputación investigadora de la universidad & 3,65 & 0,42 \\
\hline 12 & Número de alumnos por clase & 3,62 & 0,39 \\
\hline 13 & Eficacia de Bolsa de Trabajo & 3,55 & 0,32 \\
\hline 14 & Docencia y otras actividades en lengua inglesa & 3,54 & 0,31 \\
\hline
\end{tabular}




\begin{tabular}{|c|c|c|c|}
\hline 15 & Atractivo de las actividades ofertadas & 3,47 & 0,24 \\
\hline 16 & Facilidad de Financiación & 3,46 & 0,24 \\
\hline 17 & Coste anual de los estudios & 3,45 & 0,22 \\
\hline 18 & Nivel académico de los estudiantes & 3,43 & 0,20 \\
\hline 19 & Otros servicios (cafetería, informática...) & 3,43 & 0,20 \\
\hline 20 & Nivel de innovación tecnológica de la universidad & 3,43 & 0,20 \\
\hline 21 & Proporción de graduados insertados el primer año & 3,42 & 0,19 \\
\hline 22 & Recomendación familiar & 3,32 & 0,09 \\
\hline 23 & Decisión familiar & 3,28 & 0,05 \\
\hline 24 & Variedad de estudios especializados ofertados & 3,26 & 0,04 \\
\hline 25 & Proyección internacional de la Universidad & 3,24 & 0,01 \\
\hline 26 & Proporción de aprobados por curso & 3,23 & 0,01 \\
\hline 27 & Servicio de biblioteca & 3,23 & 0,01 \\
\hline 28 & Dificultad para acceder a una universidad pública & 3,22 & 0,01 \\
\hline 29 & Reputación de la Universidad & 3,19 & 0,04 \\
\hline 30 & Lengua de impartición de las clases & 3,18 & 0,04 \\
\hline 31 & Orientación humanista-integral de la formación & 3,10 & 0,13 \\
\hline 32 & Promoción e información directa a los alumnos potenciales & 3,04 & 0,19 \\
\hline 33 & Orientación religiosa & 3,03 & 0,19 \\
\hline 34 & Facilidad de acceso al transporte público & 2,98 & 0,25 \\
\hline 35 & Proximidad de la universidad al domicilio habitual & 2,89 & 0,34 \\
\hline 36 & Recomendación de un amigo & 2,80 & 0,43 \\
\hline 37 & Difusión de la universidad en los medios de comunicación & 2,79 & 0,44 \\
\hline 38 & Recomendación de un estudiante de la universidad & 2,69 & 0,54 \\
\hline 39 & Recomendación de un antiguo alumno & 2,65 & 0,58 \\
\hline 40 & Atractivo de las ofertas deportivas & 2,59 & 0,63 \\
\hline 41 & Relación con los exalumnos de la universidad & 2,59 & 0,64 \\
\hline 42 & Recomendación de un profesor & 2,55 & 0,68 \\
\hline 43 & La universidad más cercana no imparte la titulación deseada & 2,41 & 0,82 \\
\hline 44 & Posibilidad de vivir fuera del domicilio habitual & 2,29 & 0,94 \\
\hline 45 & Facilidad de acceso a la universidad & 2,23 & 0,99 \\
\hline 46 & Proximidad de la universidad a un domicilio no habitual & 2,18 & 1,04 \\
\hline 47 & Dificultad para acceder a una universidad privada & 2,10 & 1,13 \\
\hline
\end{tabular}

Fuente: Elaboración propia a partir de datos de la encuesta.

\section{CONCLUSIONES Y FUTURAS LÍNEAS DE INVESTIGACIÓN}


A partir del marco teórico sobre los factores de elección de universidad y del estudio empírico que contiene este trabajo, se derivan las siguientes conclusiones que se apuntan como orientaciones para la gestión de la citada universidad y como futuras oportunidades de investigación:

-De acuerdo con los resultados obtenidos, el hecho más remarcable es que la elección de universidad para este perfil de alumnado se fundamenta principalmente en la búsqueda de una mayor personalización de la docencia, tal como se pone de manifiesto en la valoración de los tres primeros factores de elección: Ambiente de estudio $(4,28)$, Accesibilidad del profesorado a los estudiantes $(4,08)$ y Amabilidad en el trato del personal de la universidad $(4,07)$. No obstante, la valoración que el alumnado hace sobre estos factores tiene unas condiciones de interpretación basadas a menudo en percepciones, que se pueden atribuir a diversos factores no manifestados de forma racional en la encuesta (recomendación de un profesor, tutor o amigo, por ejemplo). Ello permite justificar el carácter frecuentemente irracional de la valoración de estos atributos.

-Respecto a la valoración de los factores vinculados al profesorado, como Accesibilidad del profesorado a los estudiantes (4,08), Experiencia profesional de los profesores $(3,96)$ y Capacidades pedagógicas del profesorado $(3,83)$, insertados, según número de orden, en los lugares $2^{\circ}, 4^{\circ}$ y $7^{\circ}$, respectivamente, de la clasificación de factores de decisión para escoger centro, parece necesario tomar estos valores con precaución desde un punto de vista psicológico. ${ }^{3}$ Efectivamente, los estudiantes de bachillerato suelen tener poco claro qué son y qué implican las cualidades pedagógicas del profesorado (las pueden asociar, por ejemplo, al hecho de que éste sea "enrollado" explicando). En esta edad, es importante que el profesorado sea "accesible, amigo y si

3 A menudo, la percepción de los adolescentes sobre la calidad docente del profesorado, se basa en "el amiguismo" (CASco \& Oliva, 2005; MARTin, 2006). 
es posible, colega suyo", con independencia de sus cualidades pedagógicas e investigadoras, pues desconocen estas variables fuera de su percepción adolescente. Por tanto, los resultados que han explicitado como elementos determinantes a la hora de elegir universidad pueden no ser reales y deben tomarse con reserva en su interpretación.

-Por otra parte, se observa una moderada influencia del factor Promoción $e$ información directa a los alumnos potenciales ( $32^{\circ}$ factor de decisión), lo que parece poner de manifiesto una significativa impermeabilidad del alumnado a los instrumentos de marketing que utiliza la oferta para influir en su decisión.

-En cuanto a futuras líneas de investigación, cabe destacar la importancia de definir claramente por parte de la Universitat Abat Oliba CEU cuál es el perfil de alumno que busca y desea formar, así como los objetivos y expectativas de dicho alumnado. En cuanto al perfil de alumno que desea formar, y a partir de los resultados obtenidos, se revela necesaria una estrategia de marketing bien segmentada dada la alta relación encontrada entre el centro educativo de procedencia del alumnado y la universidad en cuestión.

-Respecto a los mecanismos de información y de promoción, las universidades deberían establecer estrategias de segmentación en función de los diferentes perfiles de los estudiantes preuniversitarios con objeto de implementar estrategias adecuadas a cada perfil. Para ello, es preciso tener en cuenta los niveles previos del sistema educativo cursado (por ejemplo, bachillerato o Ciclos Formativos de Grado Superior), la tipología de los centros de origen, el currículum académico y todas aquellas variables necesarias que permitan identificarlos y cualificarlos.

-Además, las universidades deberían implementar estrategias de comunicación complementarias a aquellas que tradicionalmente se establecen de facto dentro del 
sector educativo. Para ello, es preciso incidir de forma específica, además del destinatario final de la comunicación, es decir, el estudiante, en aquellos protagonistas que tienen capacidad de prescribir y recomendar el producto de educación superior: los stakeholders.

-La introducción del modelo de enseñanza superior basado en el Espacio Europeo de Educación Superior (EEEP) ha creado un nuevo marco de referencia en el proceso de elección de estudios y de universidad. Ello exige a las instituciones de educación superior un esfuerzo adicional en los procesos de información y promoción al alumnado para dar a conocer una oferta diferenciada.

-Finalmente, en caso de producirse progresivos descensos en la demanda universitaria, se producirá una aproximación entre patrones manifiestos e ideales. Es decir, en la medida que el número de estudiantes que quiera acceder a la universidad descienda, se puede esperar un progresivo descenso de las notas de corte en las titulaciones más presionadas en las universidades públicas $\mathrm{y}$, como consecuencia, disminuir el efecto autocensura en el proceso de manifestación de la demanda. Esta contracción de la demanda podrá afectar a toda la oferta universitaria; por tanto, en la medida que se produzca un nivel elevado de incertidumbre, se hace necesario el conocimiento de las pautas de comportamiento de la demanda universitaria con objeto de responder a sus expectativas.

\section{BIBLIOGRAFÍA}

- BELANGER, C.; MOUNT, J. \& WILSON, M. (2002) «Institutional Image and Retention». Tertiary Education and Management, Vol. 8, Nº 3, pp. 217-230. 
- BOWE, R., GEWIRTZ, S. \& BALL, S. (1994). «Capturated by the Discourse? Issues and concerns in researching (parental choice)». British Journal of Sociology of Education, vol. 15, núm. 1.

- CABRERA, A. \& LA NASA, S. (2000). «Understanding the college-choice process». En CABRERA, A. \& LA NASA, S. «Understanding the college-choice of disadvantaged students». New Directions for Institutional Research, 107, Fall 2000. Jossey Bass Publishers, pp. 5-22.

- CAÑABATE, A., (1998). La demanda de educación superior en el sistema público catalán. Un modelo estructural de previsión de la demanda universitaria pública presencial en Cataluña. [Memoria de Tesis Doctoral]. Universitat Politècnica de Catalunya, Barcelona, España.

- CASCO, F. J. \& OLIVA, A. (2005). Valores y expectativas sobre adolescentes: discrepancias entre padres, profesores, mayores y adolescentes. Infancia y aprendizaje, 28 (2), pp. 209-220.

- CUESTAS, P. J., FENOLLAR, P. \& ROMÁN, S. (2006). «Las motivaciones sociales como antecedentes del éxito académico de los estudiantes universitarios de marketing» Ponencia presentada en los XVIII Encuentros de Profesores Universitarios de Marketing, Almería, 20, 21 y 22 de Septiembre, de 2006.

- DE MIGUEL, M. \& col. (1994). El acceso a los estudios universitarios. Análisis y seguimiento de la demanda en Asturias. Madrid: Centro de Publicaciones del Ministerio de Educación y Ciencia.

- DEL OLMO ARRIAGA, J. L. (2009). Els factors d'elecció d'Universitat: el cas de la demanda a Catalunya. [memoria de Tesis Doctoral]. Universitat Abat Oliba CEU, Barcelona, España.

- FERNANDEZ LÓPEZ, S., et al. (2001). «La Demanda de Másters y Cursos de Especialización: Análisis Económico a través de un Modelo Logit Binomial» Actas de las X Jornadas de Asociación de Economía de la Educación. Universidad de Murcia. 
- FIELDER, J. S., HILTON, C. B. \& MOTES, W. H. (1993). «Educational services marketing: a proposed system for enhanced recruitment of students». Journal of Professional Services Marketing, Vol. 8, pp. 191-205.

- GAllarZA, M. G., FAYOS, T., SERVERA, D. \& ARTEAGA, F. (2008). Análisis y evaluación del servicio de formación universitaria: implicaciones para el marketing estratégico de las universidades. [web en línea]. Disponible en: <http://www.escpeap.net/conferences/marketing/ 2008_cp/Materiali/Paper/Fr/Gallarza_Fayos_Servera_Arteaga.pdf> [con acceso el 20-01-2011].

- HOLDSWORTH, D.W. \& NIND, D. (2005). «Choice Modelling New Zealand High School Seniors' Preferences for University Education». Journal of Marketing for Higher Education, Vol. 15 No. 2, pp. 81-104.

- JACKSON, G. (1986). Workable, comprehensive models of college choice. Final and technical report. US Department of Education.

- JIMÉNEZ, J. D. \& SALAS, M. (2000). «Modeling Educational Choices. A Binomial Logit Model Applied to Demand for Higher Education» Higher Education. Núm. 19 pp. 293-311.

- LOOKER, D. \& LOWE, G.S. (2001). Post-secondary access and student financial aid in Canada: Current knowledge and research gaps. [web en línea]. Disponible en: <http://www. milleniumscholarships.ca/en/foundation/ publicati ons/pareport/cprn-bkgnd.pdf> [con acceso el 13-09-2004].

- LUQUE, T. \& DEL BARRIO, S. (2007). «Análisis del valor de las percepciones de los clientes en el diagnóstico estratégico de la universidad». Ponencia presentada en el International Congress Marketing Trends, 26-27 de enero, de 2007.

- MARINGE, F. (2006). «University \& Course Choice: Implications for positioning, recruitment and marketing». International Journal of Educational Management, Vol. $20, \mathrm{~N}^{\mathrm{o}} .6$, pp. 466-479. 
- MARTIN, E. (2006). Tender puentes. Una perspectiva para educar y convivir con los adolescentes. Barcelona: Ediciones Octaedro.

- OBIS ARTAL, T \& RIALP CRIADO, J. (1996). «La Elección de una Universidad: El Caso Concreto de la Universidad Autónoma de Barcelona». Actas VIII Encuentro de Profesores Universitarios de Marketing.

- RAHONA, M. (2006). «La influencia del entorno socioeconómico en la realización de estudios universitarios: una aproximación al caso español de la década de los 90». Hacienda Pública Española/ Revista de Economía Pública. №. 178. (3/2006), pp.5580.

- SHANKA, T., QUINTAL, V. \& TAYLOR, R. (2005). «Factors influencing International Students' Choice of an Education Destination - A Correspondence Analysis». Journal of Marketing for Higher Education, Vol. 15, No. 2, pp. 31-46.

- SOUTAR, G.N. \& TURNER, J.P. (2002). «Students' preferences for university: a conjoint analysis». International Journal of Educational Management, Vol. 16, №. 1, pp. 40-45. 